University of New Hampshire

University of New Hampshire Scholars' Repository

Physics Scholarship

Physics

8-1989

\title{
Gamma-ray observations of Cygnus X-1 and Cygnus X-3 using a coded-aperture telescope
}

\author{
Mark L. McConnell \\ University of New Hampshire - Main Campus, mark.mcconnell@unh.edu \\ D J. Forrest \\ University of New Hampshire - Main Campus \\ A Owens \\ University of Leicester \\ P. P. Dunphy \\ University of New Hampshire - Main Campus \\ W T. Vestrand \\ Los Alamos National Laboratory
}

See next page for additional authors

Follow this and additional works at: https://scholars.unh.edu/physics_facpub

Part of the Astrophysics and Astronomy Commons

\section{Recommended Citation \\ M.L. McConnell, D.J. Forrest, A. Owens, P.P. Dunphy, W.T. Vestrand, and E.L. Chupp, Gamma-ray observations of Cygnus X-1 and Cygnus X-3 using a coded-aperture telescope, 1989, Ap. J., v. 343, pp. 317-322}

This Article is brought to you for free and open access by the Physics at University of New Hampshire Scholars' Repository. It has been accepted for inclusion in Physics Scholarship by an authorized administrator of University of New Hampshire Scholars' Repository. For more information, please contact Scholarly.Communication@unh.edu. 
Authors

Mark L. McConnell, D J. Forrest, A Owens, P. P. Dunphy, W T. Vestrand, and E. L. Chupp 
THE ASTROPHYSICAL JOURNAL, 343:317-322, 1989 August 1

(C) 1989. The American Astronomical Society. All rights reserved. Printed in U.S.A.

\title{
GAMMA-RAY OBSERVATIONS OF CYGNUS X-1 AND CYGNUS X-3 USING A CODED-APERTURE TELESCOPE
}

\author{
M. L. McConnell, D. J. Forrest, A. Owens, P. P. Dunphy, W. T. Vestrand and E. L. Chupp \\ Space Science Center, Institute for Earth, Oceans, and Space, University of New Hampshire \\ Received 1987 August 10; accepted 1989 January 13
}

\begin{abstract}
The Cygnus region of the sky was observed on 1984 October 1 and 2 during a balloon flight of a new $\gamma$-ray telescope. This instrument employs a coded-aperture technique to measure $\gamma$-ray photons in the energy range $160 \mathrm{keV}$ to $9.3 \mathrm{MeV}$ with an intrinsic angular resolution of 3.8. The derived spectral results for Cyg X-1 are consistent with previous measurements at energies below $\sim 1 \mathrm{MeV}$. However, in the (2-9.3) $\mathrm{MeV}$ band we find evidence for a hard spectral component with a mean flux level at the top of the atmosphere of $(7.4 \pm 2.5) \times 10^{-7}$ photons $\mathrm{cm}^{-2} \mathrm{~s}^{-1} \mathrm{keV}^{-1}$. Such emission, also suggested by some previous experiments, is not consistent with the predictions of inverse Compton models which are normally invoked to describe the $\mathrm{X}$-ray emission. It is therefore concluded that a second spectral component may be necessary to explain the present results.

Due to the large field of view (FOV) of the telescope $\left(15^{\circ} 2 \times 22^{\circ} .8\right)$, both Cyg X-1 and Cyg X-3 (with an angular separation of $\sim 8^{\circ}$ ) could be observed simultaneously. No measurable flux could be detected from Cyg $\mathrm{X}-3$, and therefore we present $1 \sigma$ upper flux limits on spectral emission from this source over the energy range $160 \mathrm{keV}$ to $9.3 \mathrm{MeV}$.
\end{abstract}

Subject headings: gamma rays: general — stars: individual (Cyg X-1, Cyg X-3) - X-rays: binaries

\section{INTRODUCTION}

Cyg X-1 was the first X-ray source discovered to be part of an optical binary system and is considered to be a prime black hole candidate. (For a general review, see Oda 1977; Liang and Nolan 1984.) This source exhibits irregular luminosity variations on all time scales from milliseconds to months. The longterm behavior can be classified into two principal states, characterized as either "high" or "low" by the relative intensity of the soft X-ray emission at energies less than $10 \mathrm{keV}$. The intensity of the soft X-ray luminosity in either of these two states is, in general, anticorrelated with the hard X-ray luminosity (Coe, Engel, and Quenby 1976; Dolan et al. 1977; Ogawara et al. 1982). However, Ling et al. (1983) have presented evidence for the existence of a third "super-low" state in which the intensities of both the hard and soft X-rays are low simultaneously. Most recently, Ling et al. (1987) have reported that the emission at energies above $300 \mathrm{keV}$ shows considerable variability within the $\mathrm{X}$-ray low state.

The X-ray emission, below a few hundred $\mathrm{keV}$, can be well understood in terms of disk accretion onto a black hole (e.g., Shapiro, Lightman, and Eardley 1976; Liang and Price 1977; Colpi, Maraschi, and Treves 1984), with the emission resulting from the Compton scattering of soft photons within a hot $\left(T_{e} \sim 10^{9} \mathrm{~K}\right)$, optically thin region of the accretion disk (Sunyaev and Titarchuk 1980). Transitions between the two metastable states of the system are often explained as resulting from some type of an instability within the accretion disk (Thorne and Price 1975; Ichimaru 1977; Lightman and Eardley 1974).

The observed X-ray spectra can be well described by an unsaturated Compton distribution with an effective temperature, $k T_{e}$, in the range of $30 \mathrm{keV}$ to $80 \mathrm{keV}$ and a Thompson optical depth, $\tau, \sim 2-5$ (e.g., Sunyaev and Trümper 1979; Nolan et al. 1981; Steinle et al. 1982; Ling et al. 1983; Perotti et al. 1986). Such spectra exhibit a steep fall off above a few hundred $\mathrm{keV}$. However, there have also been sporadic reports of excess emission in the (1-10) MeV region of the spectrum. The first such report was that of Baker et al. (1973), who presented evidence for emission in the (0.8-4.5) $\mathrm{MeV}$ band. Mandrou et al. (1978) reported measurable fluxes up to $\sim 3$ $\mathrm{MeV}$ (see also Roques et al. 1985). Ling et al. (1987) have presented evidence, based on data from the $H E A O 3$ satellite, for highly variable emission extending to at least $1.5 \mathrm{MeV}$. However, not all experiments in this energy range have provided positive results. Meegan, Fishman, and Haymes (1979) reported only very marginal results in the (1-3) MeV range. Upper limits on the $\mathrm{MeV}$ emission have been reported by Schönfelder and Lichti (1974), White et al. (1980), Nolan et al. (1981), and Nolan and Matteson (1983). The upper limits of White et al. (1980) are at a level below that of all positive measurements. At energies greater than $50 \mathrm{MeV}$, both $S A S 2$ and $\operatorname{COS} B$ failed to detect any emission from the direction of Cyg X-1 (Fichtel et al. 1975; Hermsen 1987).

Although a number of spectral measurements of Cyg X-3 have been made in the 30-200 keV range (Ulmer et al. 1974; Reppin et al. 1979; Dolan et al. 1982; Willingale, King, and Pounds 1985; Hermsen et al. 1987), only one experiment has reported detection of emission in the $200 \mathrm{keV}$ to $1 \mathrm{MeV}$ range (Meegan, Fishman, and Haymes 1979). Further observations of this source are of particular interest due to the large number of reported measurements of VHE $\left(10^{12} \mathrm{eV}\right)$ and UHE $\left(10^{15}\right.$ eV) $\gamma$-rays. (See Protheroe 1987 for a recent review of the highenergy results.)

\section{OBSERVATIONS}

The present observations were carried out using the University of New Hampshire directional gamma-ray telescope (DGT). This instrument employs the coded-aperture technique to measure celestial photons in the energy range of $160 \mathrm{keV}$ to 9.3 MeV. The intrinsic angular resolution is 3.8 over a fully 
coded field-of-view (FOV) of $15.2 \times 22: 8$. The photon detection plane is composed of a 5 by 7 array of BGO scintillation crystals, each $5.1 \mathrm{~cm}$ in diameter by $2.0 \mathrm{~cm}$ thick. The instrumental energy resolution is $19 \%$ FWHM at $662 \mathrm{keV}$. A more detailed description of the instrument may be found in Dunphy et al.(1989).

The experiment was launched from Palestine, Texas, at 14:25 UT on 1984 October 1, reaching a mean float altitude of $3 \mathrm{~g} \mathrm{~cm}^{-2}$ at 17:00 UT. The payload remained at float for $\sim 30$ hr before termination at 22:35 UT on October 2. During this time a number of sky fields were observed, including the region of the Crab Nebula and the region which includes both Cyg $\mathrm{X}-1$ and Cyg X-3. Results for the Crab region have been reported elsewhere (McConnell et al. 1987). The Cygnus region was observed from 21:30 UT on October 1 to 00:30 UT on October 2 and again from 3:30 UT to 6:00 UT on October 2, yielding a total source exposure of $3.46 \times 10^{6} \mathrm{~cm}^{2} \mathrm{~s}$ at $511 \mathrm{keV}$.

The processing of the flight data (see also Dunphy et al. 1989) involves the creation of a number of independent snapshot images obtained sequentially in time while the source region of interest is within the FOV. For the data reported here, there are 276 such "snapshots" in which both Cyg X-1 and Cyg X-3 were within the FOV. Each snapshot, representing roughly $60 \mathrm{~s}$ of instrument livetime, is first corrected for the effects of nonuniform background rates. Such background nonuniformities, if not properly handled, can lead to nonstatistical noise in the reproduced images. The procedure which is used to correct the DGT data is based on the knowledge of the relative background rates within the detector array. The relative rate of a given $\mathrm{BGO}$ detector is defined as the rate of that detector relative to the average of all 35 detectors in the detection plane. Assuming that the relative rates (expressed as factors of order unity) are constant, they can be used to correct for the inherent nonuniformities within the detector array. A review of the flight data indicates that the relative rates appear constant to a statistical level of $\sim 0.1 \%$ of the background for any given energy range, despite large variations in the overall background rate (see Fig. 1 of Dunphy et al. 1989). This gives us confidence that our correction scheme is applicable to the present dataset.

Once the snapshots are generated, they are individually projected (with 0.95 resolution) onto the celestial sphere. The sum of these projected snapshots creates a composite image which covers a large region of the sky, typically $30^{\circ} \times 30^{\circ}$. This enlarged region is caused by the motion of the FOV (with respect to the source distribution) during the observation period. This motion also results in a nonuniform exposure (i.e., effective livetime per image element) within the composite image. In the case of the Cygnus composite image, the maximum livetime per element is $\sim 13,000 \mathrm{~s}$, while the minimum is less than $100 \mathrm{~s}$ for elements near the outer edge of the image.

The composite image of the Cygnus region, compiled from the entire dataset in the energy range $200-600 \mathrm{keV}$, is shown in Figure 1. Within this image, there is clear evidence for a structure which is consistent with a source located near the position of Cyg X-1. The shape of the feature was found to be well represented by a bivariate Gaussian distribution fitted over a region roughly $10^{\circ}$ by $10^{\circ}$ in size. The measured value of the FWHM is $5.0 \pm 0.4$, which is consistent with the point spread function (PSF) measured from the Crab point source observation (McConnell et al. 1987). The upper inset of Figure 1 illustrates the $90 \%$ confidence contour for the centroid location. At
TABLE 1

Cyg X-1 and Background Count Rates For EACH OF THE ENERGY INTERVALS CONSIDERED iN THE PRESENT ANALYSIS ${ }^{\mathrm{a}}$

\begin{tabular}{ccr}
\hline $\begin{array}{c}\text { Energy } \\
(\mathrm{keV})\end{array}$ & $\begin{array}{c}\text { Background } \\
\left(\text { counts s }^{-1}\right)\end{array}$ & \multicolumn{1}{c}{$\begin{array}{c}\text { Source } \\
\text { (counts s }\end{array}$} \\
\hline $160-200 \ldots \ldots \ldots$ & 93.64 & $1.83 \pm 0.21$ \\
$200-250 \ldots \ldots \ldots$. & 85.54 & $1.50 \pm 0.24$ \\
$250-300 \ldots \ldots \ldots$. & 61.68 & $0.73 \pm 0.17$ \\
$300-400 \ldots \ldots \ldots$. & 87.11 & $1.02 \pm 0.19$ \\
$400-600 \ldots \ldots \ldots$. & 137.42 & $0.60 \pm 0.26$ \\
$600-1000 \ldots \ldots \ldots$ & 83.40 & $0.38 \pm 0.24$ \\
$1000-2000 \ldots \ldots \ldots$ & 98.36 & $-0.29 \pm 0.21$ \\
$2000-4000 \ldots \ldots \ldots$ & 35.25 & $0.16 \pm 0.11$ \\
$4000-5000 \ldots \ldots \ldots$. & 8.40 & $0.09 \pm 0.05$ \\
$5000-6000 \ldots \ldots \ldots$ & 5.92 & $0.10 \pm 0.04$ \\
$6000-9300 \ldots \ldots \ldots$ & 12.23 & $0.06 \pm 0.06$ \\
\hline a The total livetime of the measurement is 13,039 s.
\end{tabular}

the $90 \%$ confidence level, the centroid is located with an uncertainty of $\pm 16^{\prime}$. The measured location of the feature centroid is offset from the known location of Cyg X-1 by an amount which is consistent with the estimated accuracy of the pointing system $\left( \pm 30^{\prime}\right)$. We therefore conclude that the observed feature is a point source which is consistent with emission from Cyg $\mathrm{X}-1$. It is important to note that the instrument's spatial resolution is such that the emission from Cyg X-1 is clearly separated from any emission emanating from the direction of Cyg X-3, the position of which is also shown in Figure 1.

An energy-loss spectrum (see derived count rates in Table 1) was compiled from the measured response to Cyg X-1 in composite images accumulated over 11 different energy intervals. In each energy band, the response was derived using a fixed source location and PSF width as measured in the $160 \mathrm{keV}$ to $1 \mathrm{MeV}$ energy range. It is important to note that such a fitting procedure is valid in this case, since the position resolution of the discrete element BGO scintillator array (hence, the form of the PSF) is independent of energy. The statistical errors on the derived excess counts were derived using a bootstrap sampling procedure (e.g., Simpson and Mayer-Hasselwander 1986). The essential idea of the bootstrap procedure is to generate a large number of simulated datasets from the measured data. These simulated datasets, also known as bootstrap samples, are then used to estimate the statistical variability of any given parameter. In the present analysis, the simulated composite images for each energy band were generated by randomly selecting snapshot images in such a manner that the repeated selection of a given snapshot image is permitted, a process which can also be described as a random resampling with replacement. A large number of such bootstrap samples $(\sim 200)$ were used to derive the estimated distribution for the parameters of interest. A major advantage of the bootstrap process is that it evaluates parameter distributions directly from the statistical variability inherent in the data and is therefore independent of any statistical models. Errors derived from the bootstrap analysis (e.g., the source location error) have been found to be consistent with first-principle expectations (McConnell et al. 1987).

The most interesting feature of the Cyg X-1 energy-loss spectrum is the presence of positive results above $2 \mathrm{MeV}$ (four separate energy intervals with a total significance of $2.9 \sigma$ ). Such an excess was not seen during the Crab observation and is therefore considered unlikely to result from an instrumental 


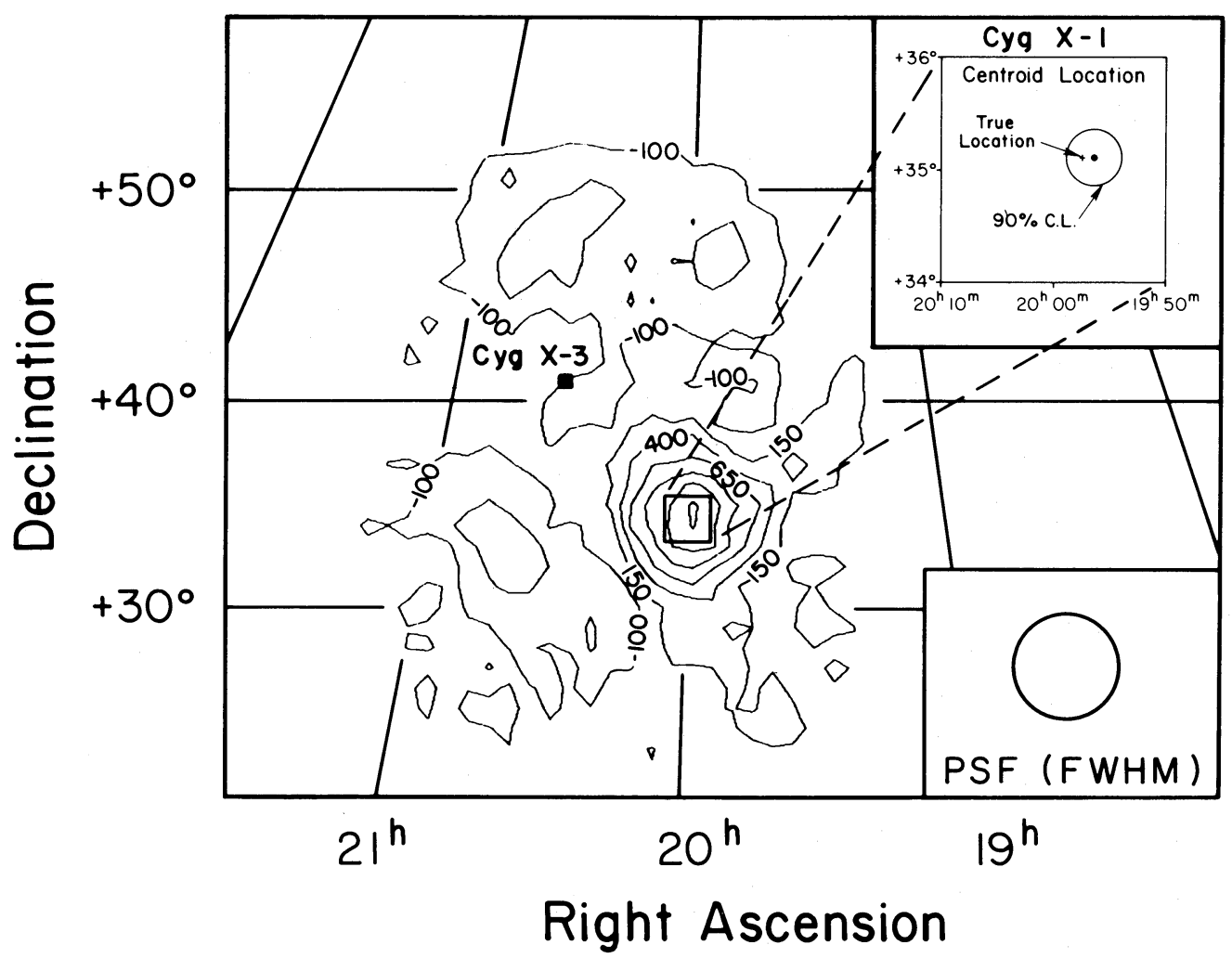

FIG. 1.-Reproduced intensity contours of the Cygnus region $\gamma$-ray emission in the energy range $200 \mathrm{keV}$ to $600 \mathrm{keV}$. The total integration time is $13,039 \mathrm{~s}$. The contour values represent the reproduced number of $\gamma$-ray events per $(0.95 \times 0.95)$ pixel, which is the convolution of the true source distribution and the point spread function (PSF) of the imaging system. Due to the nature of the image reproduction process, the average nonsource image element lies at a level less than zero ( $\sim-80$ counts; see text). The upper inset shows the $90 \%$ confidence contour for the measured peak location (as measured in the $160 \mathrm{keV}$ to $1 \mathrm{MeV}$ range). For comparison, the true location of Cyg X-1 is also shown. The location of Cyg X-3 is depicted by the solid square.

effect. Indeed, the nature of the coded-aperture process is such that the source and background measurements are made simultaneously, thus considerably reducing the possibility of instrumental background features appearing in the derived source spectrum. The data points above $2 \mathrm{MeV}$ are separated from those below $1 \mathrm{MeV}$ by a single upper limit. A very strong background line at $1.46 \mathrm{MeV}$ (due to instrumental ${ }^{40} \mathrm{~K}$ ) reduces the DGT sensitivity in the $1-2 \mathrm{MeV}$ range and may account for the lack of measurable emission.

Due to the potential importance of the results above $1 \mathrm{MeV}$, it is of interest to look at the data from a somewhat different perspective. We do this for two sample energy intervals, $200-600 \mathrm{keV}$ and 2-6 MeV, in Figures 2 and 3, respectively. In these two figures we display the frequency distribution of the amplitudes of the composite image elements for those elements having a livetime exposure greater than $10,000 \mathrm{~s}$. The amplitude of each element is expressed in terms of its statistical significance, based on a model given by Dunphy et al. (1989). We have subdivided the 435 elements which meet the livetime requirement into the 51 cells located within $4^{\circ}$ of the Cyg X-1 centroid position ("source" elements, panel $b$ ) and the remaining 384 elements outside of this region ("background" elements, panel $a$ ). The distribution of the background elements is expected to follow a Gaussian, the mean value of which should be less than zero by an amount which is directly related to the source strength (see Dunphy et al. 1989). In addition, the distribution of the source elements should follow a form which is defined by the way in which the Gaussian-shaped PSF is fit to the grid of image elements. In Figure 2, where the overall source significance is large $(8.8 \sigma)$, the difference between the source and background distributions is clearly delineated. The source cells all have positive values and are well separated from the negative mean of the background distribution. In the 2-6 $\mathrm{MeV}$ energy band (Fig. 3), the separation of the source element distribution is not as dramatic due to the lower overall significance of the source $(2.8 \sigma)$. Nevertheless, the clumping together of the source elements with an average well above the average of the background elements does demonstrate the presence of source counts in this energy band.

\section{SPECTRAL RESULTS}

There exists no unique conversion of the energy-loss data into flux units, since this conversion is dependent on the known response of the detector, the atmospheric attenuation of the source flux during the observation period, and, most importantly, the assumed source spectrum incident on the top of the atmosphere. For a given source model, a library of energy-loss spectra is generated corresponding to a range of parameters pertinent to that particular model. Comparison of the library of response spectra with the observed energy-loss spectrum then permits the determination of the best-fit model parameters, along with the statistically allowable parameter ranges.

The simplest input source model is a single power-law spectrum. The present data are marginally consistent with a power 


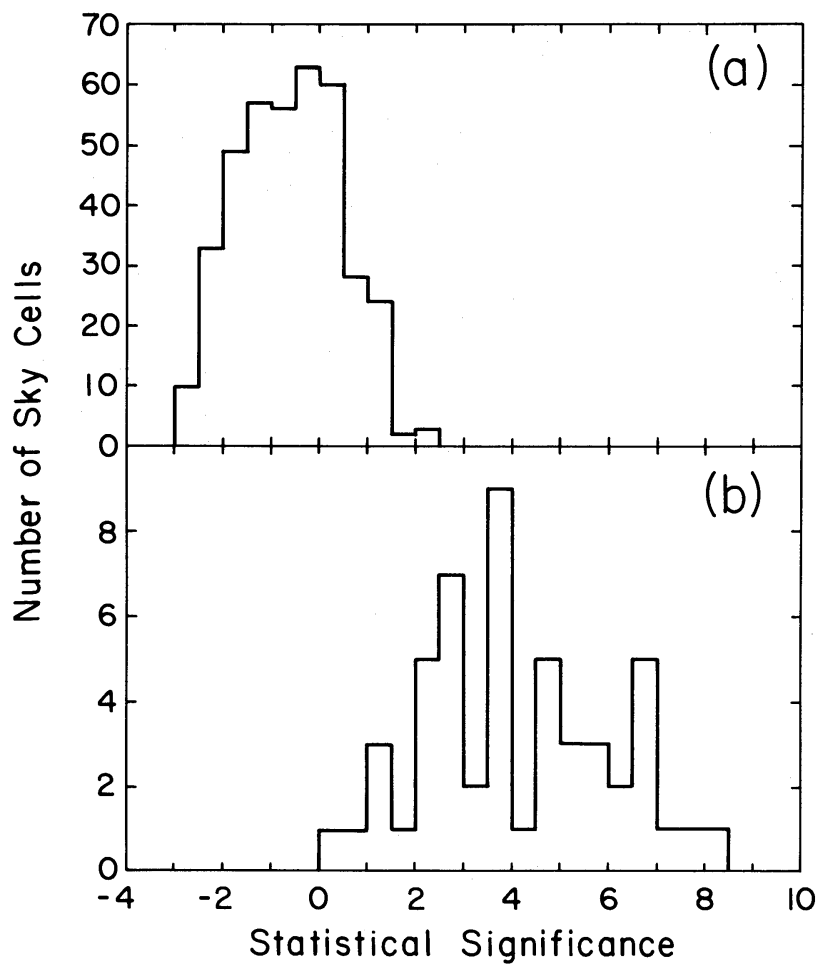

FIG. 2

Fig. 2.-The distribution of the image elements for the energy range 200-600 keV. (a) 384 sky cells having a livetime $>10,000 \mathrm{~s}$ and located more than $4^{\circ}$ from the Cyg X-1 source location. (b) 51 sky cells having a livetime $>10,000 \mathrm{~s}$ and located within $4^{\circ}$ of the Cyg X-1 source location.

law of the form $41.9 E_{\mathrm{keV}}{ }^{-2.35}$ photons $\mathrm{cm}^{-2} \mathrm{~s}^{-1} \mathrm{keV}^{-1}$. Although a best fit yielded a reduced chi-square $\left(\chi_{v}{ }^{2}\right)$ of 1.71 for 9 degrees of freedom $\left[P\left(>\chi^{2}\right)=0.08\right]$, it should be noted that the largest contribution $(\sim 54 \%)$ to the resultant $\chi^{2}$ is attributed to the data points between 4 and $6 \mathrm{MeV}$. Furthermore, when this power law is extended beyond the energy range of the present experiment, it is neither consistent with the X-ray measurements (e.g., Sunyaev and Trümper 1979) nor with the upper flux limit $>100 \mathrm{MeV}$ set by the $S A S 2$ satellite (Fichtel et al. 1975). Over a broad band, therefore, the photon spectrum cannot be characterized by a single power law.

Although a single-temperature inverse Compton model (Sunyaev and Titarchuk 1980) provides a good fit to the DGT data below $1 \mathrm{MeV}$, a fit to the entire data set yielded a relatively poor $\chi_{v}{ }^{2}$ of 1.96 for 8 degrees of freedom $\left[P\left(>\chi^{2}\right)=0.05\right]$ for best-fit parameters of $k T_{e}=177 \mathrm{keV}$ and $\tau=0.6$. Therefore, in order to derive reasonable estimates of the photon flux for the DGT data points above $1 \mathrm{MeV}$, we have followed the approach of Roques et al. (1985) and fitted these data using a single power-law model. It was found that the $1-10 \mathrm{MeV}$ data is well-described by a power law of the form $1.15 \times 10^{-4} E_{\mathrm{keV}^{-0.6}}$ photons $\mathrm{cm}^{-2} \mathrm{~s}^{-1} \mathrm{keV}^{-1}$. To obtain an acceptable fit to the entire DGT energy-loss spectrum, we have used a composite model consisting of this power-law form and an inverse Compton model. For Compton parameters of $k T_{e}=80 \mathrm{keV}$ and $\tau=2.0$, the associated $\chi_{v}{ }^{2}$ of the composite model is 1.6 for 6 degrees of freedom $\left[P\left(>\chi^{2}\right)=0.15\right]$. The photon spectrum derived using this twocomponent model is shown in Figure 4.

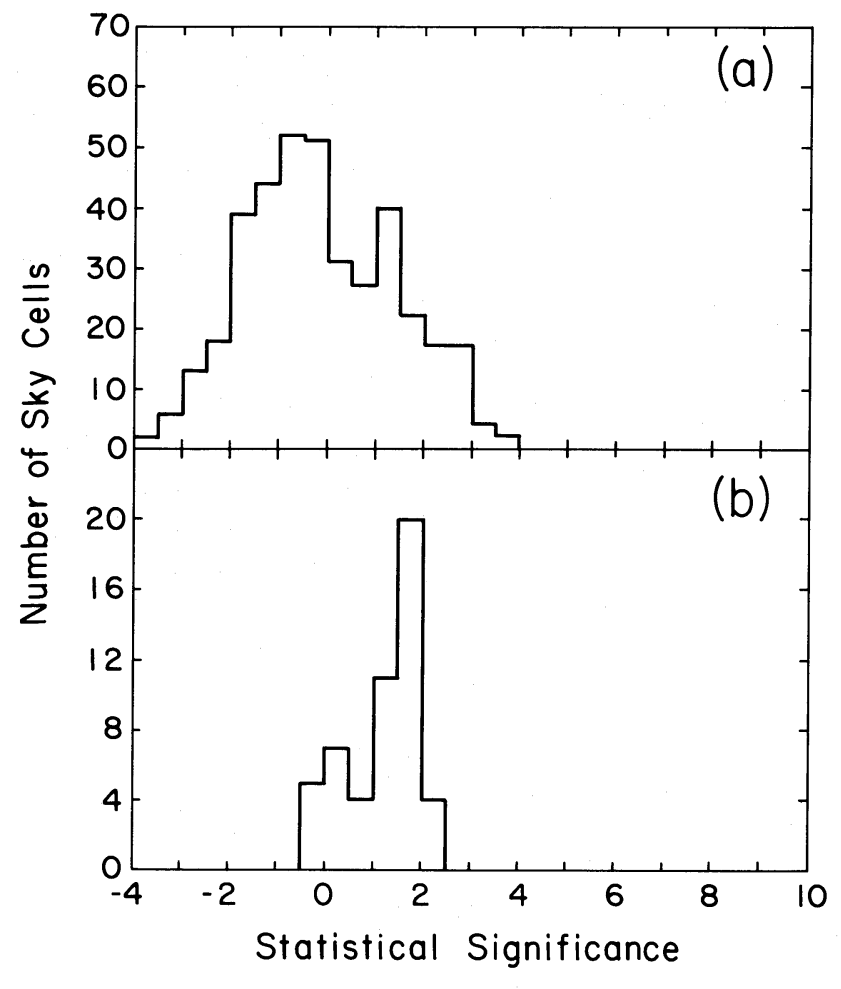

FIG. 3

FIG. 3.-The distribution of the image elements for the energy range 2-6 MeV. (a) 384 sky cells having a livetime $>10,000 \mathrm{~s}$ and located more than $4^{\circ}$ from the Cyg X-1 source location. (b) 51 sky cells having a livetime $>10,000 \mathrm{~s}$ and located within $4^{\circ}$ of the Cyg X-1 source location.

The DGT data have also been used to derive upper limits to the flux from Cyg X-3 by simultaneously fitting the imaging response of Cyg X-1 and Cyg X-3 using the measured instrumental PSF and the known location of Cyg X-3. The derived source rates are given in Table 2. The resulting upper flux limits are shown in Figure 5 along with previously reported measurements of this source. These data permit us to rule out any contamination of the Cyg X-1 results by the presence of Cyg X-3.

TABLE 2

CYG X-3 AND BACKground COUNT RATES FOR EACH OF THE ENERGY INTERVALS CONSIDERED in the Present Analysis ${ }^{\mathrm{a}}$

\begin{tabular}{ccr}
\hline \hline $\begin{array}{c}\text { Energy } \\
(\mathrm{keV})\end{array}$ & $\begin{array}{c}\text { Background } \\
\left.\text { (counts s }^{-1}\right)\end{array}$ & \multicolumn{1}{c}{$\begin{array}{c}\text { Source } \\
\text { (counts s }\end{array}$} \\
\hline $160-200 \ldots \ldots \ldots \ldots$ & 93.64 & $-0.32 \pm 0.21$ \\
$200-250 \ldots \ldots \ldots \ldots$ & 85.54 & $0.08 \pm 0.24$ \\
$250-300 \ldots \ldots \ldots$. & 61.68 & $-0.21 \pm 0.17$ \\
$300-400 \ldots \ldots \ldots \ldots$ & 87.11 & $0.16 \pm 0.19$ \\
$400-600 \ldots \ldots \ldots$. & 137.42 & $0.14 \pm 0.26$ \\
$600-1000 \ldots \ldots \ldots$. & 83.40 & $0.08 \pm 0.24$ \\
$1000-2000 \ldots \ldots \ldots$. & 98.36 & $-0.29 \pm 0.21$ \\
$2000-3000 \ldots \ldots \ldots$. & 23.16 & $-0.10 \pm 0.09$ \\
$3000-6000 \ldots \ldots \ldots$. & 26.37 & $-0.20 \pm 0.08$ \\
$6000-9300 \ldots \ldots \ldots$. & 12.23 & $0.14 \pm 0.06$ \\
\hline
\end{tabular}

a The total livetime of the measurement is $13,039 \mathrm{~s}$. 


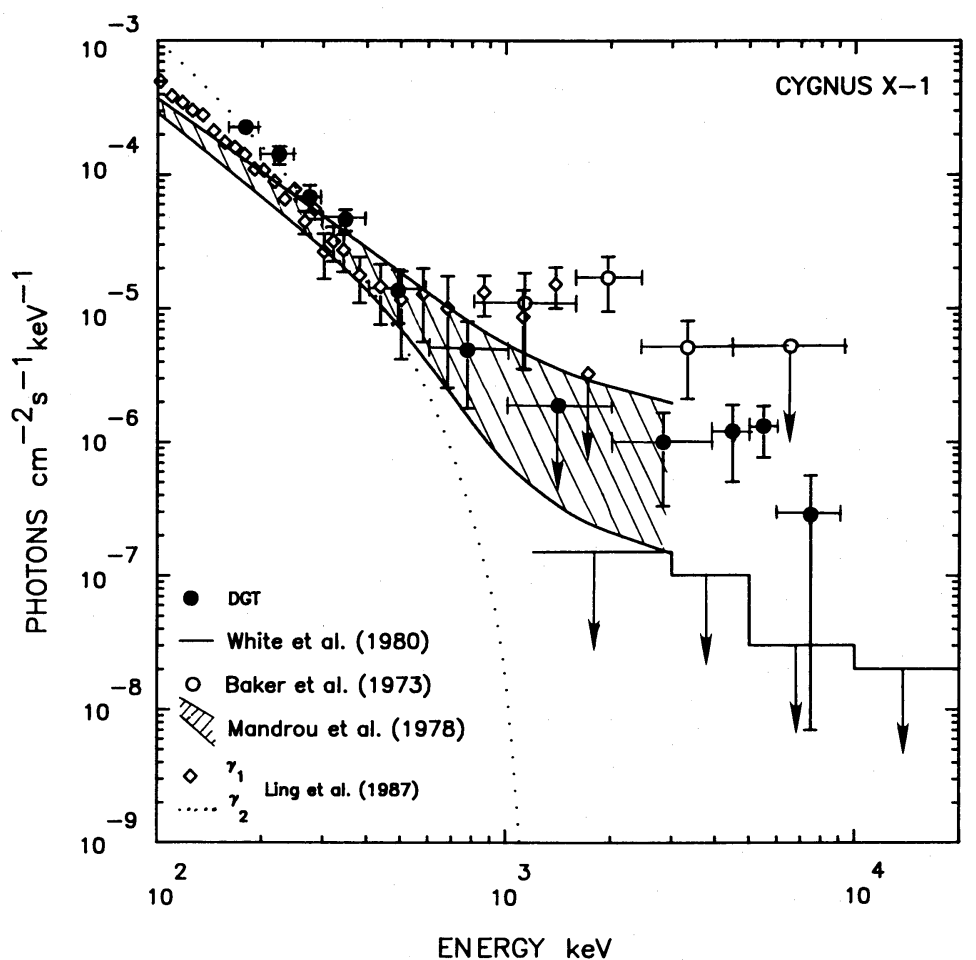

FIG. 4. - The photon spectrum of Cyg X-1, as derived from the DGT data assuming a source spectrum consisting of a power law plus Compton model (see text). Also shown are other representative observations obtained in the $1-10 \mathrm{MeV}$ energy range (with some measurements omitted for the sake of clarity). Upper limits are at the $2 \sigma$ level.

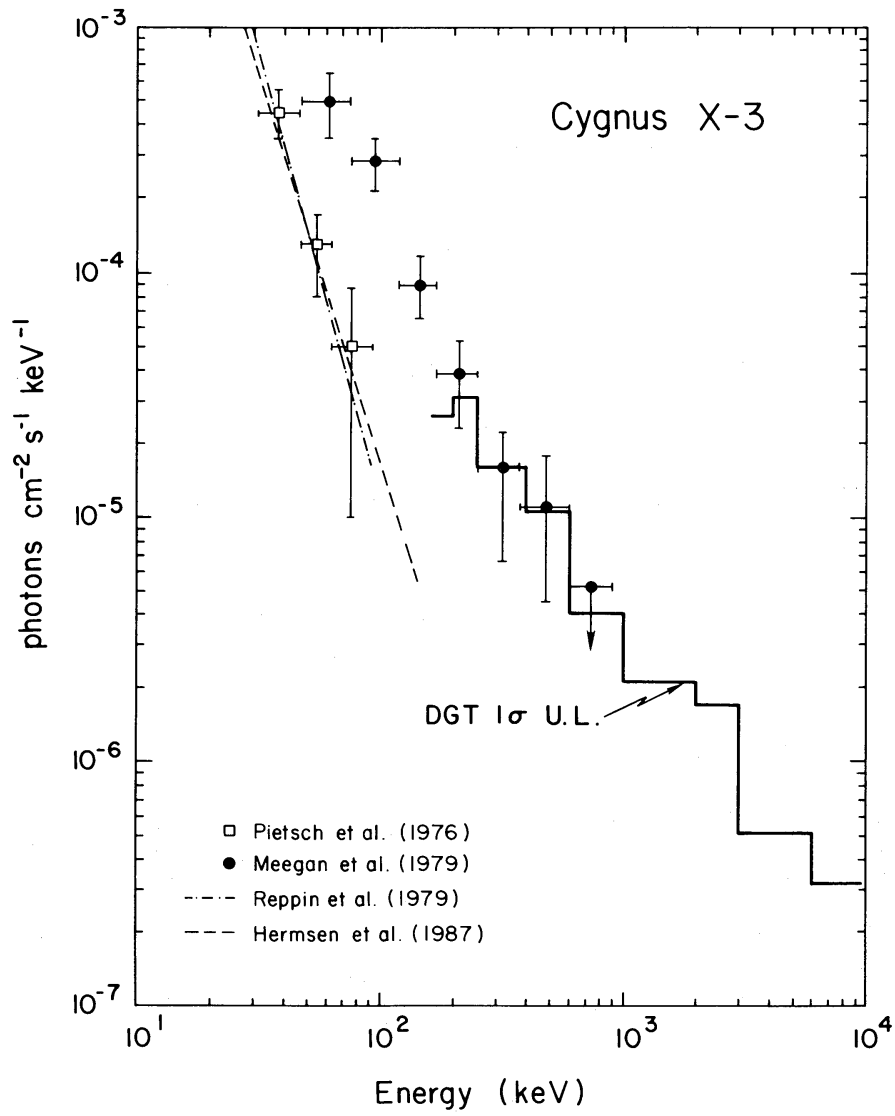

Fig. 5.-The $1 \sigma$ upper flux limits derived for the $\gamma$-ray emission from Cyg $\mathrm{X}-3$. For comparison, other representative measurements are also shown.

\section{DISCUSSION}

The present spectral results provide evidence for emission from Cyg X-1 in the $\mathrm{MeV}$ energy range. For comparison, other experimental results in the $1-10 \mathrm{MeV}$ range are shown in Figure 4. Taken collectively, these observations indicate that the $\mathrm{MeV}$ emission from Cyg X-1, if real, is variable. This conclusion is also implied by the recent $H E A O 3$ results (Ling et al. 1987). It is therefore of interest to consider the potential theoretical implications of such emission. Below, we briefly discuss the DGT results in the context of three possible thermal mechanisms: (1) a high-temperature electron-positron annihilation line (Ramaty and Meszaros 1981), (2) emission resulting from pion decay (e.g., Eilek and Kafatos 1983), and (3) nuclear line emission (e.g., Higdon and Lingenfelter 1977).

A high-temperature annihilation feature, as discussed by Ramaty and Meszaros (1981), may be modeled as a Gaussian distribution with a mean photon energy of $1.2 k T_{e}$ and FWHM of $2.6 k T_{e}$. A best fit of this model to the present data above 1 $\mathrm{MeV}$ yields an electron plasma temperature $\left(k T_{e}\right)$ of $3.5 \mathrm{MeV}$. Aside from the problems associated with making the model self-consistent (see, e.g., Lightman 1983), this temperature is clearly different from that required to explain the X-ray emission $(\sim 20-80 \mathrm{keV})$, suggesting a possibly separate emission region (Liang and Dermer 1988). This value is also much higher than the $\sim 400 \mathrm{keV}$ value suggested by the HEAO 3 results (Liang and Dermer 1988).

Some accretion disk models (e.g., Shapiro, Lightman, and Eardley 1976) postulate the existence of a two-temperature plasma in which the ion population reaches a much higher temperature $\left(T_{i} \sim 10^{12} \mathrm{~K}\right)$ than the electron population $\left(T_{e} \sim\right.$ $10^{8} \mathrm{~K}$ ). In this case, if the ion population thermalizes before reaching the event horizon, pion production may take place 
(Dahlbacka, Chapline, and Weaver 1974; Eilek 1980; Eilek and Kafatos 1983; Colpi, Maraschi, and Treves 1986). Such a process will result in the emission of $\gamma$-rays above $1 \mathrm{MeV}$ via the decay of neutral and charged pions. Based on the calculations of Eilek and Kafatos (1983), we can use the DGT results to estimate the corresponding neutral pion-decay $\gamma$-ray flux at energies greater than $100 \mathrm{MeV}$. This turns out to be $\sim 3.5 \times 10^{-3}$ photons $\mathrm{cm}^{-2} \mathrm{~s}^{-1}$, three orders of magnitude greater than the upper limit derived by $S A S 2$ (Fichtel et al. 1975). Therefore, unless the high-energy emission is time variable or degraded by high opacity, this model appears to be incapable of explaining the DGT observations.

High ion temperatures within the accretion disk may lead to nuclear line emission in the $1-10 \mathrm{MeV}$ energy range since the thermal energy of the protons in such a plasma can be well above the $\sim 10 \mathrm{MeV}$ threshold required for nuclear excitation (Higdon and Lingenfelter 1977). However, spallation processes at these temperatures would tend to remove the nuclei from the emission region, effectively suppressing line emission (Aharonian and Sunyaev 1984; Guessom and Dermer 1987; Guessom 1987). In this case, the nuclear line luminosity is estimated to be, at most, $\sim 10^{-3}$ of the total X-ray luminosity. The DGT results indicate that the luminosities above and below $1 \mathrm{MeV}$ are comparable $\left(L \sim 3 \times 10^{37} \mathrm{ergs} \mathrm{s}^{-1}\right)$. Thus it may not be possible to interpret the observed emission as nuclear line emission from a thermal plasma.

\section{SUMMARY}

We have reported on measurements of Cyg X-1 made, for the first time, with a coded-aperture telescope operating into the $\mathrm{MeV}$ region of the spectrum. This technique has made possible a clear spatial separation of any possible Cyg X-3 source contribution to the Cyg X-1 spectrum in all energy bands. The spectral results reported here for Cyg X-1 provide evidence for emission in the $2-9 \mathrm{MeV}$ region. Taken at face value, these results are difficult to explain in the context of straightforward thermal emission models.

We thank the staff of the National Scientific Balloon Facility for a successful balloon flight. Special thanks go to J. Googins for his support throughout the project. I. Gleske and M. Lauritzen were responsible for much of the design and development of the electronics. We acknowledge D. Bhattacharya and $\mathbf{M}$. Popecki for their contributions to the data analysis. M. L. M. would also like to acknowledge the support and hospitality of the Max Planck Institut für extraterrestrische Physik during the latter stages of this work. This work was supported by NASA grant NGL 30-002-021.

\section{REFERENCES}

Aharonian, F. A., and Sunyaev, R. A. 1984, M.N.R.A.S., 210, 257.

Baker, R. E., Lovett, R. R., Orford, K. J., and Ramsden, D. 1973, Nature Phys. Sci., 245, 18 .

Coe, M. J., Engel, A. R., and Quenby, J. J. 1976, Nature, 259, 544.

Colpi, M., Maraschi, L., and Treves, A. 1984, Ap. J., 280, 319. . 1986, Ap.J., 311, 150 .

Dahlbacka, G. H., Chapline, G. F., and Weaver, T. A. 1974, Nature, 250, 36.

Dolan, J. F., Crannell, C. J., Dennis, B. R., Frost, K. J., and Orwig, L. E. 1977 Nature, 267, 813 .

1982, Ap. Letters, 22, 147

Dunphy, P. P., McConnell, M. L., Owens, A., Chupp, E. L., Forrest, D. J., and

Googins, J. 1989, Nucl. Instr. Meth., A274, 362.

Eilek, J. A. 1980, Ap. J., 236, 664.

Eilek, J. A., and Kafatos, M. 1983, Ap. J., 271, 804

Fichtel, C. E., Hartman, R. C., Kniffen, D. A., Thompson, D. J., Bignami, G. F. Ögelman, H., Özel, M. E., and Tümer, T. 1975, Ap. J., 198, 163.

Guessom, N. 1987, in Nuclear Spectroscopy of Astrophysical Sources (AIP Conf. Proc. 107), ed. N. Gehrels and G. H. Share (New York: American Institute of Physics), p. 338.

Guessom, N., and Dermer, C. D. 1987, in Nuclear Spectroscopy of Astrophysical Sources (AIP Conf. Proc. 107), ed. N. Gehrels and G. H. Share (New York: American Institute of Physics), p. 332.

Hermsen, W. 1987, private communication.

Hermsen, R., et al. 1987, Astr. Ap., 175, 141.

Higdon, J. C., and Lingenfelter, R. E. 1977, Ap. J. (Letters), 215, L53.

Ichimaru, S. 1977, Ap. J., 214, 840 .

Liang, E. P., and Dermer, C. D. 1988, Ap. J. (Letters), 325, L39.

Liang, E. P., and Nolan, P. L. 1984, Space Sci. Rev., 38, 353.

Liang, E. P. T., and Price, R. H. 1977, Ap. J., 218, 247.

Lightman, A. P. 1983, in Positron-Electron Pairs in Astrophysics (AIP Conf. Proc. 101), ed. M. L. Burns, A. K. Harding, and R. Ramaty (New York: American Institute of Physics), p. 359

Lightman, A. P., and Eardley, D. M. 1974, Ap. J. (Letters), 187, L1.

Ling, J. C., Mahoney, W. A., Wheaton, W. A., Jacobsen, A. S., and Kaluzienski, L. 1983, Ap.J., 275, 307.

Ling, J. C., Mahoney, W. A., Wheaton, W. A., and Jacobson, A. S. 1987, Ap. J. (Letters), 321, L117.
Mandrou, P., Niel, M., Vedrenne, G., Dupont, A., and Hurley, K. 1978, Ap. J., 219, 288.

McConnell, M. L., Dunphy, P. P., Forrest, D. J., Chupp, E. L., and Owens, A. 1987, Ap. J., 321, 543.

Meegan, C. A., Fishman, G. J., and Haymes, R. C. 1979, Ap. J. (Letters), 234, L123.

Nolan, P. L., Gruber, D. E., Knight, F. K., Matteson, J. L., Rothschild, R. E., Marshall, F. E., Levine, A. M., and Primini, F. A. 1981, Nature, 293, 275.

Nolan, P. L., and Matteson, J. L. 1983, Ap. J., 265, 389.

Oda, M. 1977, Space Sci. Rev., 20, 757.

Ogawara Y., Mitsuda, K., Masai, K., Vallerga, J. V., Cominsky, L. R., Grunsfeld, J. M., Kruper, J. S., and Ricker, G. R. 1982, Nature, 295, 675.

Perotti, F., Della Ventura, A., Villa, G., Bassani, L., Butler, R. C., DiCocco, G., Baker, R. E., Dean, A. J., and Hanson, C. G. 1986, Ap. J., 300, 297.

Pietsch, W., Kendziorra, E., Staubert, R., and Trümper, J. 1976, Ap. J. (Letters), 203, L67.

Protheroe, R. J. 1987, Proc. 20th International Cosmic Ray Conf. (Moscow), 8, 21.

Ramaty, R., and Meszaros, P. 1981, Ap. J., 250, 384.

Reppin, C., Pietsch, W., Trümper, J., Voges, W., Kendziorra, E., and Staubert, R. 1979, Ap. J., 234, 329.

Roques, J. P., Mandrou, P., Lebrun, F., and Paul, J. 1985, Proc. 19th International Cosmic Ray Conf. (La Jolla), 1, 193.

Schönfelder, V., and Lichti, G. 1974, Ap. J. (Letters), 192, L1.

Shapiro, S. L., Lightman, A. P., and Eardley, D. M. 1976, Ap. J., 204, 187.

Simpson, G., and Mayer-Hasselwander, H. A. 1986, Astr. Ap., 162, 340.

Steinle, H., Voges, W., Pietsch, W., Reppin, C., Trümper, J., Kendziorra, E., and Staubert, R. 1982, Astr. Ap., 107, 350.

Sunayaev, R. A., and Titarchuk, L. G. 1980, Astr. Ap., 86, 121.

Sunyaev, R. A., and Trümper, J. 1979, Nature, 279, 506.

Thorne, K. S., and Price, R. H. 1975, Ap. J. (Letters), 195, L101.

Ulmer, M. P., Baity, W. A., Wheaton, W. A., and Peterson, L. A. 1974, Ap. J., 192, 691 .

White, R. S., Dayton, B., Gibbons, R., Long, J. L., Zanrosso, E. M., and Zych, A. D. 1980, Nature, 284, 608 .

Willingale, R., King, A. R., and Pounds, K. A. 1985, M.N.R.A.S., 215, 295.

E. L. Chupp, P. P. Dunphy, D. J., Forrest, M. L. McConnell, A. Owens, and W. T. Vestrand: Space Science Center, Science and Engineering Research Building, University of New Hampshire, Durham, NH 03824 